\title{
Entwicklung eines Lichen ruber unter adjuvanter IFN-alpha 2a-Therapie eines Malignen Melanoms
}

\section{Development of Lichen Ruber During Adjuvant Interferon-alpha 2a Therapy of Malignant Melanoma}

Autoren

Institute
L. Kowalzick ${ }^{1}$, L. Eickenscheidt ${ }^{1}$, F. Weiße ${ }^{2}$, J.-M. Pönnighaus ${ }^{1}$, R. A. Herbst ${ }^{2}$

Klinik für Hautkrankheiten und Allergologie, HELIOS Vogtland-Klinikum Plauen GmbH

(Ärztlicher Direktor: Prof. Dr. med. habil. L. Kowalzick)

2 Klinik für Hautkrankheiten und Allergologie (Chefarzt: Prof. Dr. med. R. A. Herbst), HELIOS Klinikum Erfurt GmbH (Ärztlicher Direktor: Prof. Dr. med. D. Esser)

\section{Bibliografie}

Dol http://dx.doi.org/

10.1055/s-0029-1214900

Online-Publikation: 2. 7. 2009

Akt Dermatol 2010; 36:

88-90 ๑ Georg Thieme

Verlag KG Stuttgart · New York ISSN 0340-2541

\section{Korrespondenzadresse}

Prof. Dr. med. habil.

Lutz Kowalzick

Klinik für Hautkrankheiten und Allergologie

HELIOS Vogtland-Klinikum

Plauen $\mathrm{GmbH}$

Postfach 100153

08505 Plauen

lutz.kowalzick@

helios-kliniken.de

\section{Zusammenfassung \\ $\nabla$}

Wir berichten über einen 73-jährigen Patienten, bei dem eine adjuvante Low-dose-Therapie mit $3 \times$ wöchentlich $3 \mathrm{Mio}$. IE Interferon-alpha 2a (Roferon $A^{\circledR}$ ) s.c. wegen eines zwei Monate zuvor außerhalb primär und nachexzidierten Malignen Melanoms Stadium IIb begonnen wurde. Etwa drei Monate nach Beginn der Interferontherapie kam es zum Auftreten eines exanthematischen Li-

\section{Einleitung \\ $\nabla$}

Der Lichen ruber (planus) (LR) ist eine subakute bis chronisch entzündliche papulöse Dermatose mit typischer Klinik und Histologie unbekannter Genese. Eine immunologische hypergische Reaktion gegen unbekannte Antigene auf Keratinozyten, die teilweise solchen auf viral induziert veränderten Hepatozyten gleichen könnten, wird als möglicher Pathomechanismus vermutet [1]. Eine erhöhte Prävalenz von $6 \%$ bei Patienten mit Hepatits-C-Virus (HCV)-Infektion wurde berichtet. Diese erhöht sich auf $17 \%$ bei Patienten unter Interferon (IFN)-Therapie dieser Infektion $[2,3]$. Dagegen wurde nur in Einzelfällen das Auftreten eines LR unter IFN-Therapie ohne gleichzeitige HCV-Infektion beobachtet [4].

Wir berichten hier über einen HCV-negativen Patienten mit einem Malignen Melanom im Stadium IIb, bei dem drei Monate nach Beginn einer adjuvanten Low-dose-Interferon-Therapie mit IFN-alpha 2a (Roferon $A^{\circledR}$ ) ein exanthematischer LRP des oberen Stammes auftrat.

\section{Kasuistik}

Wir berichten über einen 73-jährigen Patienten, bei dem eine adjuvante Low-dose-Therapie mit $3 \times$ wöchentlich 3 Mio. IE IFN-alpha 2a (Roferon chen ruber am oberen Stamm, der histologisch gesichert wurde. Serologisch konnte eine frühere oder bestehende Hepatitis B- oder C-Virus-Infektion ausgeschlossen werden. Der vorgestellte Fall ist unseres Wissens der erste exanthematische und der erste unter einem Low-dose-Schema beschriebene Fall von Lichen ruber während adjuvanter Interferon-Therapie bei einem Patienten mit Malignem Melanom.

$\left.A^{\circledR}\right)$ s.c. wegen eines zwei Monate zuvor außerhalb primär und nachexzidierten Malignen Melanoms Stadium IIb begonnen wurde. Der Tumor war am linken Unterarm lokalisiert, die Tumordicke nach Breslow betrug 2,6 mm mit Mikroulzeration, der Invasionslevel nach Clark wurde mit IV angegeben. Eine Sentinel-Lymphknotenbiopsie wurde wegen des zu großen Zeitabstandes zur Exzision und des großen Sicherheitabstandes von allseits $2 \mathrm{~cm}$ nicht mehr durchgeführt.

Etwa drei Monate nach Beginn der IFN-Terapie kam es zum exanthematischen Auftreten juckender monomorpher erythematöser etwas hyperkeratotischer Papeln von bis zu $1 \mathrm{~mm}$ Durchmesser am vorderen und hinteren oberen Stamm. Einige einzelne Herde wiesen eine spiegelnde Oberfläche auf oder waren angedeutet polygonal $(\bullet$ Abb. 1). Das sonstige Integument und die Übergangsschleimhäute fanden sich frei.

Auch zum Ausschluss einer Psoriasis punctata, deren Auftreten unter einer IFN-Therapie nicht ungewöhnlich wäre [5], sowie eines Morbus Darier führten wir eine Probebiopsie vom oberen Rücken durch. Die Epidermis fand sich unregelmäßig verschmälert und von sägezahnartiger Konfiguration mit Hyperorthokeratose. Das Stratum granulosum fand sich teilweise prominent, das Stratum basale fast vollständig aufgehoben mit zahlreichen Zytoidbodys. Im angrenzenden Korium stellte sich ein lockeres bandförmiges 


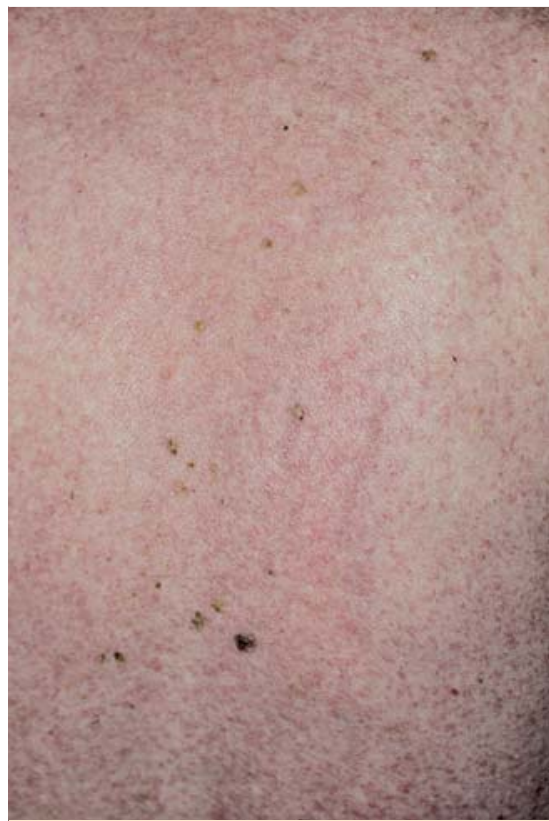

Abb. 1 Lichen ruber exanthematicus, der sich bei einem 73-jährigen Patienten ca. 3 Monate nach Beginn einer adjuvanten Low-dose-IFN-alpha 2aTherapie wegen eines Malignen Melanoms entwickelte.

Im Bereich der oberen Flanke finden sich disseminiert multiple erythematöse hyperkeratotische Papeln von meist unter $1 \mathrm{~mm}$ Durchmesser, z. T. mit spiegelnder Oberfläche und angedeutet polygonaler Konfiguration. Nebenbefundlich finden sich multiple, typische bräunliche und hyperkeratotisch breit aufsitzende seborrhoische Keratosen mit bis zu $1 \mathrm{~cm}$ Durchmesser.

lichenoides lymphohistiozytäres Infiltrat sowie Pigmentinkontinenz dar ( $\bullet$ Abb. 2). Zusammenfassend wurde die histologische Diagnose eines teilweise atrophen Lichen ruber gestellt.

Serologisch konnte eine frühere bzw. bestehende Hepatitis A-, Boder C-Virus-Infektion ausgeschlossen werde (HAV-IgM-, HBcIgM, HCV-Antikörper, HBs-Antigen jeweils negativ).

Die Hauterscheinungen sprachen unter fortgesetzter adjuvanter IFN-Therapie des Malignen Melanoms auf topische Kortikosteroide über neun Monate kaum an. Bei Staging-Untersuchungen in dreimonatigen Abständen fand sich kein Anhalt für ein Rezidiv des Malignen Melanoms.

\section{Diskussion}

Das Neuauftreten eines LR unter einer IFN-Therapie wurde mit Ausnahme von Patienten, die diese wegen einer Virus-Hepatitis erhielten, nur in wenigen Fällen mit meist hämatologischen Neoplasien berichtet. Unseres Wissens ist bislang nur ein einziger Fall eines LR unter adjuvanter IFN-Therapie eines Malignen Melanoms beschrieben worden. Dieser Patient erhielt wegen einer axillären Lymphknotenmetastasierung (Stadium III) nach operativer Ausräumung eine IFN-Hochdosistherapie nach Kirkwood mit IFN-alpha $2 b$ (Intron $A^{\circledR}$ ) in einer Dosis von 10 Mio. IE fünf bzw. dreimal wöchentlich. Drei Monate nach Therapiebeginn entwickelte der Patient disseminierte pruritische erythematöse Papeln an den Handwurzelgelenken, den Unterarmen und Fußrücken sowie eine retikuläre weißliche Zeichnung der Wangenschleimhaut. Die Diagnose eines LR wurde histologisch gesichert. Virusserologien (HBV, HCV, EBV, CMV) waren negativ. Unter einer systemischen Kortikosteroidtherapie kam es trotz fortgesetzter IFN-Therapie zu einer weitgehenden Besserung der Hautveränderungen. Trotz planmäßiger Beendigung der HochdosisIFN-Therapie nach 12 Monaten Dauer kam es danach noch zum

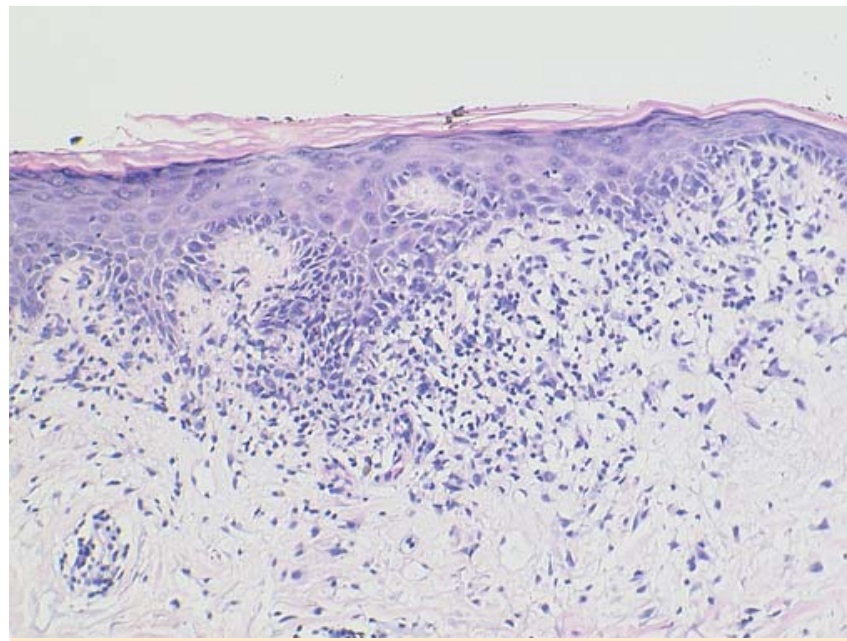

Abb. 2 Histologisches Präparat (HE-Färbung, 100-fache Vergrößerung) von klinisch typischem Herd: Die Epidermis findet sich unregelmäßig verschmälert und von sägezahnartiger Konfiguration mit Hyperorthokeratose. Das Stratum granulosum ist teilweise prominent, das Stratum basale fast vollständig aufgehoben mit zahlreichen Zytoidbodys. Im angrenzenden Korium stellt sich ein lockeres bandförmiges lichenoides lymphohistiozytäres Infiltrat sowie Pigmentinkontinenz dar. Diagnose: teilweise atropher Lichen ruber.

Neuauftreten einzelner, weitgehend asymptomatischer LR-Herde [4].

Unser Fall stellt unseres Wissens den ersten publizerten Fall des Neuauftretens eines LR unter einer adjuvanten Low-dose-IFNTherapie wegen Malignen Melanoms dar. Das erstmalige Auftreten der LR-Hautveränderung lag ebenfalls etwa 3 Monate nach Therapiebeginn. Im Unterschied zum vorbeschriebenen Fall fand sich jetzt keine klassische Lokalisierung der LR-Herde an den Prädilektionsstellen, sondern ein exanthematischer Befall des oberen Stamms. Eine Schleimhautbeteiligung fand sich ebenfalls abweichend hiervon in unserem Fall nicht.

Eine topische Kortikosteroidtherapie erwies sich in unserem Fall als wenig effektiv, während systemische Steroide im vorbeschrieben Fall - zumindest vorübergehend - gut wirksam waren. Wir verzichteten aber wegen der Tumorerkrankung auf eine systemische immunsuppressive oder eine PUVA-Therapie. Auf eine systemische Retinoidtherapie verzichteten wir wegen Leberenzymerhöhungen unter der IFN-Therapie.

Das Neuauftreten oder die Exazerbation von vermutlich teilweise autoimmunologisch induzierten entzündlichen Hauterkrankungen wie Psoriasis vulgaris [6] und Vitiligo [7] unter adjuvanter IFN-Therapie des Malignen Melanoms ist bekannt und nicht selten. Dagegen erscheint die Induktion eines LR hierunter ein deutlich selteneres Ereignis zu sein, an das jedoch gedacht werden sollte und das gegebenenfalls durch eine Probebiopsie gesichert bzw. ausgeschlossen werden sollte.

\section{Abstract}

\section{Development of Lichen Ruber During Adjuvant Interferon-alpha 2a Therapy of Malignant Melanoma \\ $\nabla$}

We report on a 73-year-old male caucasian patient who received an adjuvant low-dose treatment with s.c. 3 MU interferon-alpha 2a (Roferon $A^{\circledR}$ ) three times a week because of a stage IIB malignant melanoma excised in two sessions with security margins 
two months ago. About three months after the beginning of the interferon therapy the patient developed an exanthematic lichen ruber which diagnosis was secured by histopathology. A former or present Hepatitis B- or C-virus infection could be excluded by serology. The present case is to the best of our knowledge the first described which developed during an adjuvant low-dose interferon regimen because of malignant melanoma and the first exanthematic case during an interferon therapy for that indication.

\section{Literatur}

1 Vogt T. Papulöse und lichenoide Erkrankungen. In: Braun-Falco $O$ et al. (Hrsg). Dermatologie und Venerologie. 5. Aufl. Heidelberg: Springer, 2005: $498-593$

2 Agner T, Fogh $H$, Weissmann $K$. The relation between lichen planus and hepatitis C: A case report. Acta Derm Venereol 1992; 72: 380

3 Sánchez-Pérez J, de Castro M, Buezo GF et al. Lichen planus and hepatitis $C$ virus: Prevalence and clinical presentation of patients with lichen planus and hepatitis C virus infection. Br J Dermatol 1996; 134: 715 719

4 Saval AH, Martinez FC. Lichen planus induced by interferon- $\alpha-2 B$ therapy in a patient with cutaneous malignant melanoma. Acta Derm Venereol 1999; 79: 395

5 Asnis LA, Gaspari AA. Cutaneous reactions to recombinant cytokine therapy. J Am Acad Dermatol 1995; 33: 393-410

6 Hartmann F, von Wussow P, Deicher H. Psoriasis-Exazerbation bei Therapie mit $\alpha$-Interferon. Dtsch Med Wochenschr. 1989; 20: 96 - 98

7 Le Gal FA, Paul C, Chemaly P, Dubertret L. More on cutaneous reactions to recombinant cytokine therapy. J Am Acad Dermatol 1996; 35 : 50 651 